\title{
Evaluating Access Mechanisms for Multimodal Representations of Lifelogs
}

\author{
Zhengwei Qiu, Cathal Gurrin, and Alan F. Smeaton \\ Insight Centre for Data Analytics, \\ Dublin City University, Dublin 9, Ireland \\ \{zhengwei.qiu, cathal.gurrin, alan.smeaton\}@dcu.ie
}

\begin{abstract}
Lifelogging, the automatic and ambient capture of daily life activities into a digital archive called a lifelog, is an increasingly popular activity with a wide range of applications areas including medical (memory support), behavioural science (analysis of quality of life), workrelated (auto-recording of tasks) and more. In this paper we focus on lifelogging where there is sometimes a need to re-find something from one's past, recent or distant, from the lifelog. To be effective, a lifelog should be accessible across a variety of access devices. In the work reported here we create eight lifelogging interfaces and evaluate their effectiveness on three access devices; laptop, smartphone and e-book reader, for a searching task. Based on tests with 16 users, we identify which of the eight interfaces are most effective for each access device in a knownitem search task through the lifelog, for both the lifelog owner, and for other searchers. Our results are important in suggesting ways in which personal lifelogs can be most effectively used and accessed.
\end{abstract}

Keywords: lifelogging, HCI, multimodal access

\section{Introduction}

Continuing advances in technology have led us to the point where lifelogging, the recording of the totality of life experience using digital sensors, is an activity that anyone can engage in [11]. In selecting an appropriate definition of lifelogging, we use the description by Dodge and Kitchin [5] and define it as "a form of pervasive computing, consisting of a unified digital record of the totality of an individual's experiences, captured multi-modally using digital sensors, stored permanently as a personal multimedia archive and made accessible in a pervasive manner through appropriate interfaces". Lifelogging can offer societal benefits in applications like memory support, diet monitoring, quality of life analysis, self-reflection, monitoring progress of degenerative conditions, and more, as well as auto-recording of work-related tasks. Yet in each application, the benefits can only be realised if the lifelog content can be easily and efficiently accessed.

Heretofore, lifelog access has typically based on a browsing metaphor whereby data/time, geo-location, or content filters support visual browsing of a lifelog, which has been typically structured into events. There has been little consideration of how people might search a lifelog across a variety of devices at any 
time and in any place [11]. It is our conjecture that to be truly effective, a lifelog needs to support ubiquitous access from multiple devices, for both browsing and search tasks. Hence, when designing lifelogging tools, we believe that appropriate content modeling and representation techniques should be applied to lifelogs in order to support multi-modal access. In this work we develop a state-of-the-art holistic lifelogging solution covering capture, analysis, indexing, browsing and search. We present a first multi-modal access model for lifelog data and we consider its impact in terms of the time to find a known item across three popular access devices, smartphone, laptop and ebook reader, using eight different user interface types.

\section{Background to Lifelogging and Pervasive Access}

Lifelogging has been the subject of research since Mann's early work on wearable computing culminating in his CHI paper from a decade ago [17], which focused mainly on data gathering. The seminal MyLifeBits project [9] from Microsoft Research created the first database of life data from Gordon Bell's life and was one of the motivating factors in the development of the first accessible visual lifelogging device, the Microsoft SenseCam. This is a wearable camera that can automatically take up to 5,000 images from the wearer's viewpoint in a single day. It resulted in a host of lifelogging research that explored issues of data organisation for lifelogs such as how to segment a sequence of images from a day into a series of events and represent them in a storyboard manner [15] on a desktop system. Nowadays we find lifelogging applications and interfaces running on many mobile devices and even TVs [12]. At the same time as the work on general visual lifelogs, there have been domain-focussed applications of lifelogging, such as Aizawa's Foodlog [1], or any of the current generation of commercial lifelogging devices from OMG or Narrative. More recently, there have been efforts at using the smartphone as a lifelogging platform [4], which has shown to be as effective as a dedicated device such as a SenseCam [10]. In all of the above work, there is one lifelog interface running on one device. However, if lifelogging is to be a pervasive activity with anytime access as per the definition of lifelogging that we adopt, then there needs to be consideration of how to access a lifelog from across multiple devices.

Before we even begin to consider user interaction, lifelogging solutions require a certain level of basic data organisation or pre-processing, such as segmenting a day's activities into events or perhaps annotating data [11]. Studies on autobiographical memory have suggested that it is part of our nature to organise our daily experiences into events. Prior research has shown that appropriate annotations of lifelog events can significantly increase the success rate for locating known items from a lifelog [6]. Once an appropriate event segmentation model has been identified, then the challenge of how to efficiently access a lifelog needs to be considered [2] [22]. Automatic or manual annotation of events in lifelogs makes them more accessible for either searching or browsing tasks [7] and this is something we need to consider when designing lifelog access tools. 
With regard to multi-modal access, Hess et al. investigated user requirements and preferences for multiple-device media systems [13] arguing that lifelog content needs to be shared and accessed across personal and shared devices. Fleury et al. report an approach to transform video content from a mobile device to a television [8] and point out that more attention is needed to consider differences among devices such as size of screen and keyboard availability. Ubiquitous computing scenarios not only enable ubiquitous access, but also bring a challenge for system and user interface designers. As it becomes increasingly difficult to optimally represent content across the myriad of devices currently available due to different sizes, resolutions, interaction mechanisms and environments of use, Human-Computer Interaction (HCI) experts have realised the importance of characteristics of output devices on interface design [19]. We see that different products representing different paradigms require different solutions for accessing [21]. Since lifelog content is media-rich, it can naturally be presented in different ways and since there is no single best way to present lifelog data on every device, the best is to use different representations on different devices for different usage scenarios. That is the main point addressed in this paper.

\section{An End-to-End Holistic Lifelogging Solution}

In order to explore the effectiveness of different approaches to multimodal lifelog access covering both searching and browsing tasks, one needs an end-to-end lifelogging solution, from capture to access, and to evaluate this with users and real-world lifelog data. To this end, we applied state-of-the-art components to develop an holistic lifelogging process as illustrated in Figure 1.

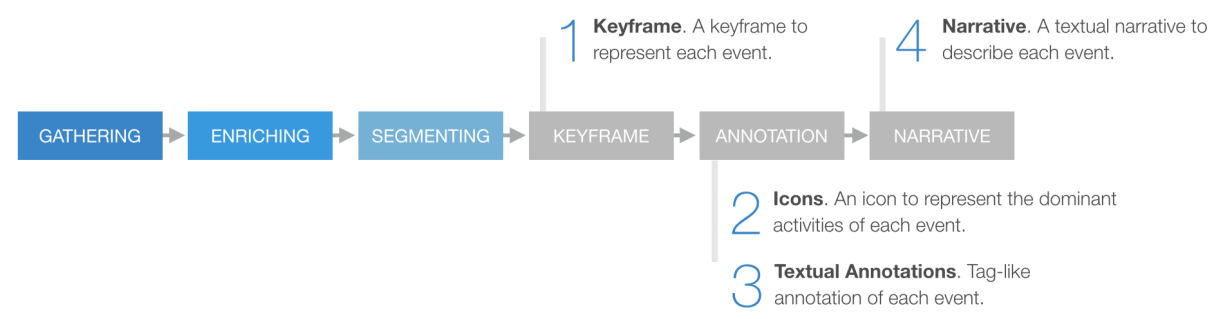

Fig. 1: Components of an holistic lifelogging process, from gathering data, through enriching, segmenting, keyframe extraction, semantic annotation and narrative generation.

We summarise the six core components of our holistic lifelogging process (as shown in Figure 1):

- Gathering. The lifelog is gathered using a smartphone running our inhouse developed lifelogging software [18]. Sensed data from the smartphone includes auto-captured images, location (using GPS, WIFI and base station), 
music listened to, photos viewed, environmental (sound levels, weather), communications (phone-call and SMS logs) and data from other sensors (accelerometer, in-range Bluetooth devices, screen, etc..).

- Enriching. Images gathered are annotated with metadata from the smartphone based on temporal alignment of captured data. This occurs in a semantic enrichment step in which the smartphone's sensor data is semantically enriched using empirically observed rules, machine learning techniques and an analysis of the statistical frequency and (re)occurrence of the sensed data. This produces a set of six 'contexts' forming the semantic enrichment. These are physical and social locations (e.g. addresses and meaningful descriptions such as home, work, etc.), social relationships \& interactions (e.g. friends, family, stranger identification), actual and relative time (where relative time refers to concepts such as yesterday, last week, etc.), environmental factors at the time of capture such as weather, the physical and semantic activities of the wearer (e.g. sitting, running, using the phone, working, etc), and a personal profile of the lifelogger that understands where home is and who people are.

- Segmenting. As with most work on visual lifelogging, an algorithm for event segmentation is used to segment each day's data into a list of discrete events based on analysing data for context changes, a typical approach in lifelogging [3]. We employ a Support Vector Machine (SVM) in the event segmentation process and consider a wider range of source features than has been done in prior work, including image, location, activity, social and environment attributes. We employed attributes extracted from raw sensor data instead of using raw sensor data itself, such as the similarity of adjacent images, semantic locations and activity changes. We evaluated the performance of this event segmentation algorithm and found that $77.4 \%$ of 2,470 event boundaries (on the experiment dataset described later) were detected correctly vs. a manual ground truth, $22.6 \%$ were false negatives and $0.7 \%$ false positive instances which are not actually event boundaries but which were detected as such. This accuracy is similar though not significantly better than the state-of-the-art and gives a suitable platform to base our work on. The end result is that a lifelog becomes a series of events, each represented by a set of temporally organised images and metadata.

- Keyframe. To represent each event in a lifelog, a single keyframe is usually identified and used [11]. Our approach for identifying keyframes is built on two concepts, social and quality; if some photographs contain faces, choose the best quality ones as the keyframe. If no face is detected in the event, choose the best quality photograph as the keyframe.

- Annotation. Each event is annotated automatically to produce both an iconic representation of the dominant activity of the event as well as a textual annotation of the dominant activities, concepts and contexts of the event.

- Narrative. Finally, each event is represented by a narrative description of the event, similar to a diary entry. The process by which narrative is generated is a three-part process (namely fabula, sjuzet and discourse generation). Fabula is a series of sentences based on the detected contexts and segmented 
events, generated from the second annotation phase; sjuzet is a paragraph of narratives generated from the fabula without the repeated sentences; and discourse is a paragraph of narrative with an illustrated picture/keyframe taken during the event.

Based on this lifelogging framework we are able to create four individual semantic elements of lifelog representation for multimodal access, as shown in Figure 1. These represent the ways in which people communicate information in a static manner namely keyframes in storyboards, icons in storyboards, tag annotations and textual narratives, each of which we now describe.

1. Keyframes. The single keyframe used to represent each event is chosen as described. This allows a storyboard to be generated for each day in a lifelog and allows a user to scan a series of events based on the keyframe alone and make a decision as to whether that event may contain a known item/activity in which the user is interested.

2. Icons. Based on the set of identified activities within a given event, an icon is used to represent the dominant activity in that event. We focus on a single icon to represent each event on the assumption that multiple icons might be confusing. In total, we identified fifteen possible activities, based on the semantic lifestyle activities identified in [14]. The icons include activities such as working, eating, commuting, and relaxing, and could be considered to cover the majority of the range of daily activities. This representation allows a user to scan a series of events on a device that may not have the high resolution expected for keyframes or even screens which render colour.

3. Textual Annotations. Here we use a tag-like annotation to represent each event generated based on the context and activities of that event. This is in the form of a set of tags that describe the context of the event and on average, five such tags were generated for each event.

4. Narrative. A narrative description that represents the context and activities of an event is the final representation format. This can be seen as a more natural description of the event, as one might create for a diary entry. An example of a narrative description for an event would be "It was 10am and you were working at your computer with Frank for one hour". The narrative text is automatically generated based on the annotations.

\section{Modeling for Multimodal access}

Based on the four semantic elements described earlier, we explored eight different interfaces to accessing a lifelog. These were interfaces that had been previously built and deployed, for example storyboards of event keyframes (with and without annotations) and animated sequences. Where the access device allowed, all keyframe-based interfaces supported drill-down analysis to show all images that are associated with the event. In addition, we designed new interfaces inspired by diaries and information visualisation, such as storyboards of icons and textual narratives (with and without keyframes). This resulted in the creation of the eight different lifelog interface types outlined below: 


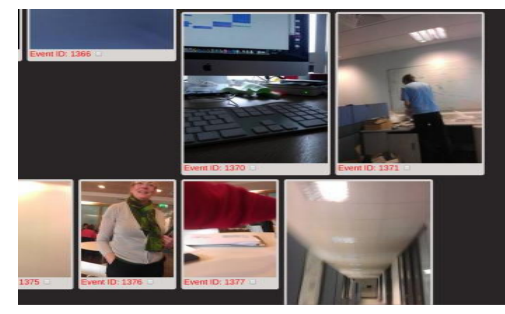

(a) Keyframes

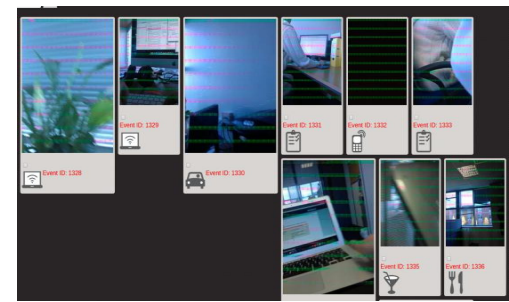

(c) Keyframes \& Icons

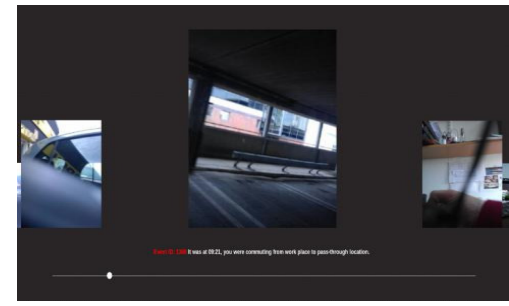

(e) Animations

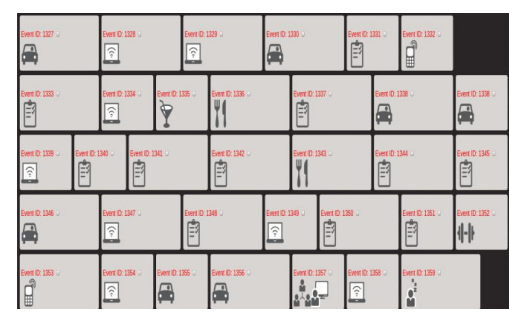

(g) Icons

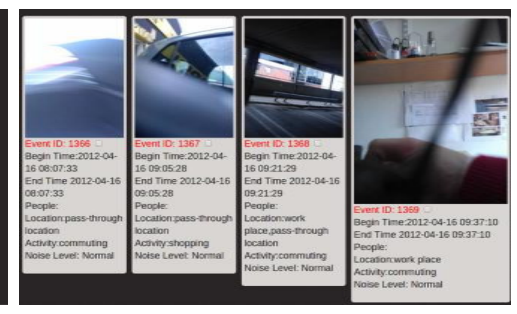

(b) Keyframes \& Annotations

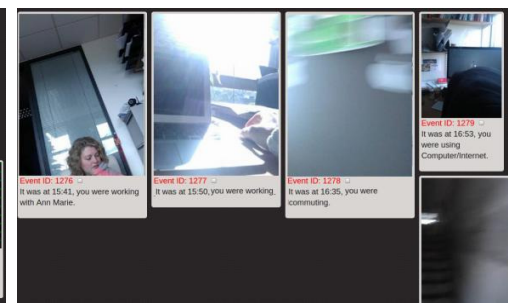

(d) Keyframes \& Narratives

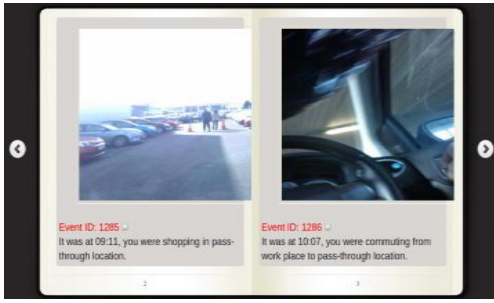

(f) Visual Diaries

Fig. 2: User interfaces employed in this research

- Keyframes: A traditional lifelogging interface in which all events in a day are shown on a single webpage, as in Figure 2a with events presented chronologically. This is our baseline interface and is based on the storyboard interface used in [15]. 
- Keyframes \& Annotations: Adding textual annotations underneath each keyframe, as in Figure 2b; this represents a minimal addition to the baseline keyframe-only interface.

- Keyframes \& Icons: Users' activities are represented by icons in addition to keyframes, as in Figure 2c. The icon provides a quick reference visual cue to represent the 15 semantic life activities previously mentioned.

- Keyframes \& Narratives: Containing keyframes and associated textual narratives, as shown in Figure 2d.

- Animations: This shows all of a day's events as keyframes with related narrative in animation mode from beginning to end of the day. The time span between showing two events is $500 \mathrm{~ms}$. The interface is shown in Figure $2 \mathrm{e}$ and would be useful in a less-interactive, lean-back environment, such as on a TV or other relaxation-focused device and is based on the original SenseCam player [11].

- Visual Diaries: Keyframes and related narratives are shown in a diary-style storybook, as shown in Figure 2f. The user can turn pages of events as if using an e-book reader.

- Icons: A storyboard of icons only, as shown in Figure 2g. The icon list provides a small visual summary of an event for a display suitable for small screen devices, such as Google Glass or Apple watch.

- Narrative Diaries: A storyboard of event narrative descriptions, as shown in Figure 2h. This narrative description replicates the concept of a written diary and could be used to summarise days, weeks or longer time periods in a simple textual form.
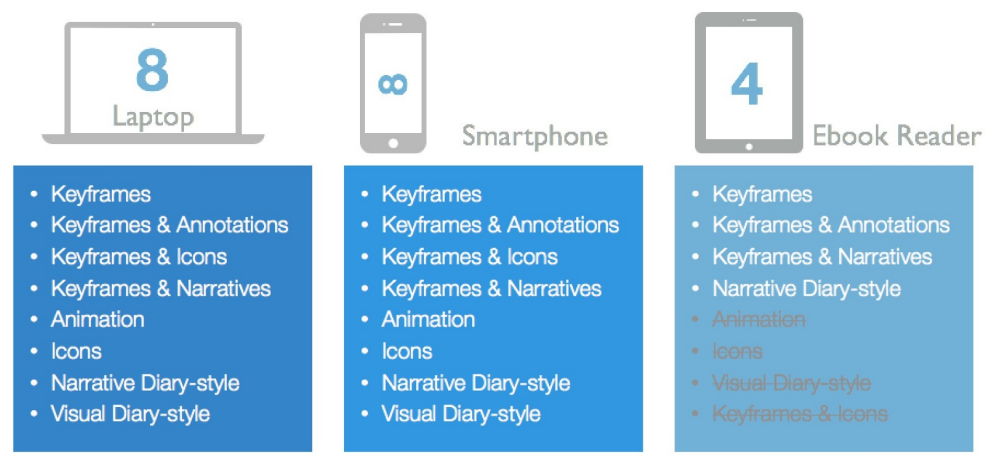

Fig. 3: Mapping Interfaces to Devices

These eight interfaces were mapped to three physical access devices for our evaluation. The devices chosen were the laptop computer, smartphone and ebook reader. Each of these has different user access characteristics. The laptop represents the conventional lifelog access device and can support detailed user interactions using the large screen and trackpad. The smartphone (4-inch Android 
smartphone) integrates touch-screen access on a small screen and represents today's handheld computing devices. Finally the e-book reader represents a diary for replicating how people have traditionally logged and recalled their memories and while it can display images, it is monochrome and there is limited potential for user interaction, except for turning pages.

For both the laptop and smartphone, we evaluated all eight interfaces. However, for the e-book reader, we evaluated only four of the interfaces due to limitations of the device and the lack of clarity on-screen when we have a complex interface display. Hence we focused on "Keyframes", "Keyframes \& Annotations", "Keyframes \& Narratives" and "Narrative Diary" on the e-book reader and we generated different PDF files to represent these interfaces.

\section{User Evaluation}

In order to evaluate which types of lifelog visualisations and representations are most suitable for display on different devices, we recruited one lifelogger (data gatherer and query generator) and 16 participants to evaluate the interfaces, 11 male and 5 female. Each works in, or have studied in a university, are from a broad range of disciplines, all are computer literate and would have computers/laptops and most have used e-book readers previously. All evaluators were given basic background knowledge about the lifelogger, but they were not told who the lifelogger was. In this way, any effects of some users knowing the lifelogger's lifestyle better than others were thus avoided.

\subsection{Experimental Dataset}

We generated a ten-day lifelog dataset of all-day lifelog use from the lifelogger (referred to as LL). This lifelog data was imported and enriched by the lifelogging process described earlier and the access interfaces were generated. From the ten days' data, the event segmentation technique identified 253 events and automatically selected appropriate keyframes. Accompanying the data, LL generated 16 information needs from the ten day lifelog, including queries, descriptions and correct answers, that could be identified by an independent third-party user. Information needs such as "How many times was LL using the computer on the 20th ?", "Find all the events involving working with AM on the 9th April" and "At what time was LL driving to work on the 18th?".

\subsection{The Interface Evaluation Process}

For the evaluation, eight subjects evaluated the laptop-based interface, four evaluated the mobile interface and four evaluated the e-book interface. Interfaces were presented to subjects in a controlled order to avoid bias. For the laptopbased interface, each user attempted eight topics (one for each interface), meaning that every topic was evaluated 4 times. For the mobile and e-book interfaces, the subjects evaluated all sixteen topics across the 8 and 4 interfaces respectively. 


\begin{tabular}{|c|c|c|c|c|c|c|c|c|}
\hline Device & 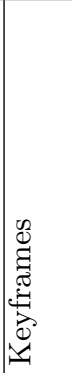 & 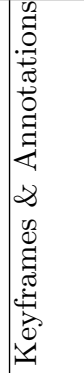 & 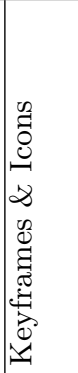 & 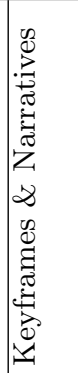 & 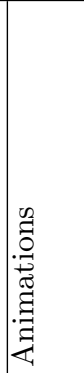 & 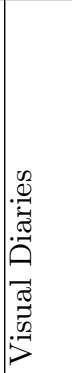 & 告 & 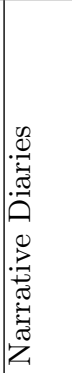 \\
\hline Laptop & 7.4 & 11.5 & 12.3 & 9.8 & 11.6 & 14.4 & 13.0 & 16.0 \\
\hline Phone & 23.4 & 29.2 & 21.5 & 23.4 & 32.3 & 46.6 & 28.7 & 23.4 \\
\hline $\begin{array}{l}\text { E- } \\
\text { book }\end{array}$ & 94.6 & 73 & - & 52.6 & - & - & - & 47.5 \\
\hline
\end{tabular}

Table 1: Average time to completion (in seconds) for each interface.

Immediately following the evaluation, subjects completed a survey aimed at identifying their qualitative opinions about each interface. As a reminder, for each user interface, the participant was presented with a screenshot of the user interface. A seven-point Likert scale was employed and the questionnaire took approximately 5-10 minutes to complete and all users completed the questionnaire without errors. This survey included four aspects: visual appeal, subjective satisfaction, potential for errors and speed of use. Visual appeal is believed to dominate impression judgments [16]. The other three aspects (effectiveness, efficiency and satisfaction) are defined by the international standard ISO/IEC 9241-11 and have been extensively employed in interface evaluation [20].

\subsection{Results of the Interface Evaluation}

Beginning with a quantitative analysis and the seek time to find the event of interest, the laptop computer has been shown to be the fastest approach to access information in personal lifelogs (as shown in Table 1). It took participants only 12 seconds on average to find the answer on the computer. On the smartphone and E-book reader it took 29 and 73 seconds respectively. This is not unexpected since both the smartphone and the e-book have interface limitations. Interestingly, the slowest laptop interface was faster than the best smartphone interface, and similar when comparing the smartphone and e-book reader. As can be seen from Table 1, keyframe-based interfaces were the best-performing on the laptop, with the narrative diaries performing slowest. On the smartphone, the best-performing interfaces were also keyframe based. For the e-book reader, the best-performing interfaces by a significant amount of time were narrative based, with narrative alone being the fastest. We understand that this is due to the limitations of the display technology of a monochrome e-book reader and we noted that in the PDF conversion, the images were compressed heavily, further hindering any image-based interface.

With regard to the qualitative user survey, on the laptop, the subjects preferred the Visual-Diary Style interface in terms of subjective satisfaction and 


\begin{tabular}{|c|c|c|}
\hline & $\begin{array}{l}\text { Optional } \\
\text { interface }\end{array}$ & Reasons to use \\
\hline \multirow[t]{2}{*}{$\begin{array}{l}\text { Top } 2 \text { interfaces } \\
2^{*} \text { for Computer }\end{array}$} & Visual-Diary Style & $\begin{array}{l}\text { Highest visual appeal and subjec- } \\
\text { tive satisfaction, lowest error poten- } \\
\text { tial }\end{array}$ \\
\hline & Keyframes & Fastest for laptop users. \\
\hline \multirow[t]{2}{*}{$\begin{array}{l}\text { Top } 2 \text { interfaces } \\
2^{*} \text { for Smartphone }\end{array}$} & Keyframes \& icons & Highest accuracy, fastest UI. \\
\hline & Animations & $\begin{array}{l}\text { Highest visual appeal and subjec- } \\
\text { tive satisfaction. }\end{array}$ \\
\hline \multirow[t]{2}{*}{$\begin{array}{l}\text { Top } 2 \text { interfaces } \\
2 * \text { for E-book reader }\end{array}$} & Narrative Diary Style & $\begin{array}{l}\text { The fastest, most accurate, highest } \\
\text { visual appeal and subjective satis- } \\
\text { faction, lowest potential for errors. }\end{array}$ \\
\hline & Keyframes and Narratives & $\begin{array}{l}\text { The second fastest, second most ac- } \\
\text { curate, second highest visual appeal } \\
\text { and subjective satisfaction, second } \\
\text { lowest potential for errors. }\end{array}$ \\
\hline
\end{tabular}

Table 2: The two suggested user interfaces for different devices.

visual appeal. For the smartphone, the preferred interface was keyframes and icons because of its high accuracy and speed of use. Finally, due to the characteristics of the e-book reader, Narratives was selected as the preferred interface for E-book reader, because it was fastest, most accurate, highest visual appeal and subjective satisfaction, lowest potential for errors.

Based on these results, we propose the top two interfaces for each access device in Table 2. For the laptop, we suggest that a Keyframes storyboard helps locate content quickly, but that users enjoy the slower Visual-Diary style most. However, Keyframes \& Icons provided a visually appealing and reasonably fast interface that provides a trade-off. For the smartphone, Keyframes \& Icons would be the fastest interface, though in terms of user-appeal, the significantly slower animation interface would be best. The trade-off in this case is Keyframes \& Annotations, which provided reasonable speed and user satisfaction. Finally, for the e-book reader, Narratives has shown great potential in helping users to access their lifelog data using an E-book reader, hence this is our recommendation for E-book reader users. Given the visual limitations of the e-book reader, a second option would be merging narratives with keyframes, which was reasonably fast, but also had high visual appeal and subjective satisfaction.

\section{Discussion}

To be effectively exploited and used across a range of usage scenarios, a personal lifelog needs to be accessible across a variety of access devices and not just from a laptop or from a mobile device. In this work we created eight lifelogging interfaces using state-of-the-art components and evaluated their effectiveness using three access devices on a holistic lifelogging framework. We identified (unsurprisingly) 
that the laptop is significantly faster than the other devices, with the e-book reader the slowest by far. For each device, we identified the fastest interface for users to browse for a 'known item' and identified the interfaces that the experimental subjects found most appealing.

Naturally there are some limitations of this study. Our users set were small and all were university-based and very computer-literate. We employed a small test collection and any browsing mechanism is likely to fail when a user is faced with months or years of lifelog data. In such cases, a search mechanism is required. It is our belief that many of the preliminary findings in this paper will translate into a search mechanism also. In addition, like all other work in personal lifelogging it is difficult to make our data collection openly available for use by others because of the very personal nature of the data itself, and this prevents the kind of collaborative benchmarking exercises seen in TREC. However, this is the first such study which evaluates lifelog access and will provide valuable clues for lifelog designers in future work. Finally, there are new ubiquitous-access devices, such as Google Glass and Apple watch which represent other potential interfaces to evaluate and these will be the subject of future experimentation.

\section{Acknowledgements}

This paper is based on research conducted with financial support of Science Foundation Ireland under grant number SFI/12/RC/2289.

\section{References}

1. K. Aizawa, Y. Maruyama, H. Li, and C. Morikawa. Food balance estimation by using personal dietary tendencies in a multimedia food log. Multimedia, IEEE Transactions on, 15(8):2176-2185, Dec 2013.

2. K. Aizawa, D. Tancharoen, S. Kawasaki, and T. Yamasaki. Efficient retrieval of life log based on context and content. In Proceedings of the the 1st ACM workshop on Continuous archival and retrieval of personal experiences, pages 22-31, 2004.

3. G. Chen and D. Kotz. A survey of context-aware mobile computing research. Technical report, Technical Report TR2000-381, Dept. of Computer Science, Dartmouth College, 2000.

4. D. De Jager, A. L. Wood, G. V. Merrett, B. M. Al-Hashimi, K. O'Hara, N. R. Shadbolt, and W. Hall. A low-power, distributed, pervasive healthcare system for supporting memory. In Proceedings of the First ACM MobiHoc Workshop on Pervasive Wireless Healthcare, page 5. ACM, may 2011.

5. M. Dodge and R. Kitchin. Outlines of a world coming into existence: Pervasive computing and the ethics of forgetting. Env. and Plan. B, 34(3):431-445, 2007.

6. A. R. Doherty, C. Gurrin, and A. F. Smeaton. An investigation into event decay from large personal media archives. In EIMM 2009 1st ACM International Workshop on Events in Multimedia, pages 49-56, October 2009.

7. A. R. Doherty, K. Pauly-Takacs, N. Caprani, C. Gurrin, C. J. A. Moulin, N. E. O'Connor, and A. F. Smeaton. Experiences of Aiding Autobiographical Memory Using the SenseCam. Human-Computer Interaction, 27(1-2):151-174, 2012. 
8. A. Fleury, J. S. Pedersen, and L. Bo Larsen. Evaluating user preferences for video transfer methods from a mobile device to a TV screen. Pervasive and Mobile Computing, 9(2):228-241, 2013.

9. J. Gemmell, G. Bell, and R. Lueder. MyLifeBits: a personal database for everything. Communications of the ACM, 49(1):88-95, 2006.

10. C. Gurrin, Z. Qiu, M. Hughes, N. Caprani, A. R. Doherty, S. E. Hodges, and A. F. Smeaton. The SmartPhone as a Platform for Wearable Cameras in Preventative Medicine. Am J Prev Med, 44(3):308-313, Mar 2013.

11. C. Gurrin, A. F. Smeaton, and A. R. Doherty. LifeLogging: Personal Big Data. Foundations and Trends in Information Retrieval, 8(1):1-125, 2014.

12. C. Gurrin, Z. Zhang, H. Lee, N. Caprani, D. Carthy, and N. E. O'Connor. Gesturebased personal archive browsing in a lean-back environment. In MMM 2010 - The 16th International Conference on Multimedia Modelling, January 2010.

13. J. Hess, B. Ley, C. Ogonowski, L. Wan, and V. Wulf. Jumping between devices and services: towards an integrated concept for social TV. In Proceddings of the 9th International Interactive Conference on Interactive Television, pages 11-20. ACM, 2011.

14. D. Kahneman, A. B. Krueger, D. A. Schkade, N. Schwarz, and A. A. Stone. A survey method for characterizing daily life experience: The day reconstruction method. Science, 306:1776-1780, 2004.

15. H. Lee, A. F. Smeaton, N. E. O'Connor, G. J. Jones, M. Blighe, D. Byrne, A. Doherty, and C. Gurrin. Constructing a SenseCam Visual Diary as a Media Process Multimedia Systems. Multimedia Systems Journal, 14(6):341-349, 2008.

16. G. Lindgaard, C. Dudek, D. Sen, L. Sumegi, and P. Noonan. An exploration of relations between visual appeal, trustworthiness and perceived usability of homepages. ACM Transactions on Computer-Human Interaction (TOCHI), 18(1):1, 2011.

17. S. Mann, J. Fung, C. Aimone, A. Sehgal, and D. Chen. Designing EyeTap Digital Eyeglasses for Continuous Lifelong Capture and Sharing of Personal Experiences. In Alt.CHI, Proceedings of CHI, 2005.

18. Z. Qiu, C. Gurrin, A. Doherty, and A. F. Smeaton. A Real-Time life experience logging tool. Advances in Multimedia Modeling, pages 636-638, 2012.

19. S. Robertson, C. Wharton, C. Ashworth, and M. Franzke. Dual device user interface design: PDAs and interactive television. In Proceedings of the SIGCHI conference on Human factors in computing systems, pages 79-86. ACM, 1996.

20. B. Shneiderman. Designing the user interface: strategies for effective humancomputer interaction, volume 2. Addison-Wesley Reading, MA, 1992.

21. M. Tambe. TV human interface: Different paradigm from that of PC and Mobile. IEEE Code of Ethics, 2012.

22. D. Tancharoen, T. Yamasaki, and K. Aizawa. Practical life log video indexing based on content and context. In Electronic Imaging 2006, pages 60730E-60730E. International Society for Optics and Photonics, 2006. 